

\title{
Dynamics of System of Higher Order Difference Equations with Quadratic Terms
}

\author{
Erkan Taşdemir \\ Pınarhisar Vocational School, Kırklareli University, 39300, Kırklareli, Turkey \\ erkantasdemir@hotmail.com \\ Abstract
}

This paper aims to investigate the global asymptotic stability of following system of higher order difference equations with quadratic terms:

$$
x_{n+1}=A+B \frac{y_{n}}{y_{n-m}^{2}}, y_{n+1}=A+B \frac{x_{n}}{x_{n-m}^{2}}
$$

where $A$ and $B$ are positive numbers and the initial values are positive numbers. We also study the rate of convergence and oscillation behaviour of the solutions of related system.

Keywords: Difference equations; global asymptotic stability; boundedness; rate of convergence; oscillation

\section{Introduction}

Nowadays, investigation of dynamic behaviour of recursive sequences (also called difference equations) have attracted significant interest. This attention concern with applications to different fields of sciences. Applied sciences need many mathematical models. Mathematical models easily can be create from difference equations or their systems. Although the appearance of the difference equations is simple, the solutions of these equations are difficult to understand and are different from each other. Particularly since the solutions of the higher order difference equations differ in every order, it becomes difficult to understand the behavior of their solutions. Especially researchers have studied the global asymptotic stability, boundedness and oscillatory behavior of system of difference equations. There are many examples related to difference equations or systems. Therefore, studies on difference equations are increasing day by day and will continue to increase. There are many articles and books in the literature on the theory of difference equations and systems, see [1]-[26]. Additionally, there are many papers related to our study as follows:

In [6], Camouzis et al investigated the dynamics of solutions of following system of difference equations

$$
x_{n+1}=1+\frac{x_{n}}{y_{n-m}}, y_{n+1}=1+\frac{y_{n}}{x_{n-m}} .
$$

They especially studied the global asymptotic behavior of solutions of the related system.

In [19], Papaschinopoulos et al dealt with the oscillation, the bounded solutions, and the 
global asymptotic stability of the system of difference equations

$$
x_{n+1}=A+\frac{y_{n}}{x_{n-q}}, y_{n+1}=A+\frac{x_{n}}{y_{n-q}} .
$$

In [26], Zhang et al handled the boundedness, persistence and global asymptotic stability of solutions of system of difference equations

$$
x_{n+1}=A+\frac{y_{n-m}}{x_{n}}, y_{n+1}=A+\frac{x_{n-m}}{y_{n}} .
$$

In [25], Zhang et al studied the dynamics of the following symmetrical system of rational difference equation

$$
x_{n+1}=A+\frac{y_{n-k}}{y_{n}}, y_{n+1}=A+\frac{x_{n-k}}{x_{n}} .
$$

Additionally, in [10], Gümüş dealt with the global asymptotic stability and rate of convergence of related system.

In [3], Beso et al investigated the boundedness and global asymptotic stability of solutions of following difference equation

$$
x_{n+1}=\gamma+\delta \frac{x_{n}}{x_{n-1}^{2}} .
$$

In [23], Taşdemir investigated the oscillation, global asymptotic stability and rate of convergence of solutions of following system of second order difference equations with quadratic terms

$$
x_{n+1}=A+B \frac{y_{n}}{y_{n-1}^{2}}, y_{n+1}=A+B \frac{x_{n}}{x_{n-1}^{2}} .
$$

In this paper, we study the system of higher order difference equations with quadratic terms

$$
x_{n+1}=A+B \frac{y_{n}}{y_{n-m}^{2}}, y_{n+1}=A+B \frac{x_{n}}{x_{n-m}^{2}}
$$

where $A$ and $B$ are positive numbers and the initial values are positive numbers and $m \in\{2,3, \cdots\}$. We especially investigate the stability, global behaviour and rate of convergence of solutions of system (1). We also study the oscillation behaviour of solutions of related system.

Now, we give three theorems which are used during this study.

Theorem 1 (Linearized Stability Theorem [16], p.11) Assume that

$$
X_{n+1}=F\left(X_{n}\right), n=0,1, \cdots,
$$

is a system of difference equations such that $\bar{X}$ is a fixed point of $F$.

(i) If all eigenvalues of the Jacobian matrix B about $\bar{X}$ lie inside the open unit disk $|\lambda|<1$, that is, if all of them have absolute value less than one, then $\bar{X}$ is locally asymptotically stable.

(ii) If at least one of them has a modulus greater than one, then $\bar{X}$ is unstable.

Theorem 2 ([4]) Let $n \in N_{n_{0}}^{+}$and $g(n, u, v)$ be a decreasing function in $u$ and $v$ for any 
fixed $n$. Suppose that for $n \geq n_{0}$, the inqualities

$$
\begin{aligned}
& y_{n+1} \leq g\left(n, y_{n}, y_{n-1}\right) \\
& u_{n+1} \geq g\left(n, y_{n}, y_{n-1}\right)
\end{aligned}
$$

hold. Then

implies that

$$
y_{n_{0}-1} \leq u_{n_{0}-1}, y_{n_{0}} \leq u_{n_{0}}
$$

$$
y_{n} \leq u_{n}, n \geq n_{0}
$$

Let us consider the following system of difference equations:

$$
E_{n+1}=(A+B(n)) E_{n}
$$

where $E_{n}$ is a $k$-dimensional vector, $A \in C^{k \times k}$ is a constant matrix, and $B: \mathbb{Z}^{+} \rightarrow C^{k \times k}$ is a matrix function satisfying

$$
\|B(n)\| \rightarrow 0
$$

as $n \rightarrow \infty$, where $\|\cdot\|$ denotes any matrix norm that is associated with the vector norm

$$
\|(x, y)\|=\sqrt{x^{2}+y^{2}}
$$

Theorem 3 (Perron's Theorem, [20]) Assume that condition (3) holds. If $E_{n}$ is a solution of (2), then either $E_{n}=0$ for all $n \rightarrow \infty$, or

or

$$
\lim _{n \rightarrow \infty} \sqrt[n]{\left\|E_{n}\right\|}
$$

$$
\lim _{n \rightarrow \infty} \frac{\left\|E_{n+1}\right\|}{\left\|E_{n}\right\|}
$$

exists and is equal to modulus of one of the eigenvalues of matrix $A$.

\section{Main Results}

In this here, we firstly apply the following the change of the variables for system (1):

Hence we obtain the new system as:

$$
t_{n}=\frac{x_{n}}{A}, z_{n}=\frac{y_{n}}{A}
$$

$$
t_{n+1}=1+p \frac{z_{n}}{z_{n-m}^{2}}, z_{n+1}=1+p \frac{t_{n}}{t_{n-m}^{2}}
$$

where $p=\frac{B}{A^{2}}>0$. From here on, we consider the system (4).

Now we examine the equilibrium point of system (4).

Lemma 4 Let $p>0$. The system (4) has a unique positive equilibrium point such as

$$
(\bar{t}, \bar{z})=\left(\frac{1+\sqrt{1+4 p}}{2}, \frac{1+\sqrt{1+4 p}}{2}\right) .
$$




\subsection{Boundedness of system (4)}

This section, we determine the boundedness character of system (4).

Lemma 5 Assume that $\left\{\left(t_{n}, z_{n}\right)\right\}$ be a positive solution of system (4) and $p>0$. Then $t_{n}>1$ and $z_{n}>1$ for $n \geq 1$.

Proof. Let $\left\{\left(t_{n}, z_{n}\right)\right\}$ be a positive solution of system (4). Hence we obtain from system (4):

$$
\begin{aligned}
& t_{1}=1+p \frac{z_{0}}{z_{-m}^{2}}>1 \\
& z_{1}=1+p \frac{t_{0}}{t_{-m}^{2}}>1 .
\end{aligned}
$$

Thus, we have the followings by induction

$$
\begin{aligned}
& t_{n+1}=1+p \frac{z_{n}}{z_{n-m}^{2}}>1, \\
& z_{n+1}=1+p \frac{t_{n}}{t_{n-m}^{2}}>1 .
\end{aligned}
$$

Therefore, the system (4) has a bound from below as desired.

Theorem 6 Suppose that $0<p<1$. Therefore every solutions of system (4) have a bound from above.

Proof. Now we know from Lemma 5 that $t_{n}>1$ and $z_{n}>1$ for $n \geq 1$ and $p>0$. Otherwise, every solutions of system (4) satisfies

$$
t_{n+1}<1+p+p^{2} t_{n-1}, n \geq 1 .
$$

We consider Theorem 2. According to this, $t_{n} \leq u_{n}, n=0,1, \ldots$, where $\left\{u_{n}\right\}$ satisfy

$$
u_{n+1}=1+p+p^{2} u_{n-1}, n \geq 1
$$

such that

Therefore the solution $u_{n}$ of the difference equation (6) is

$$
u_{s}=t_{s}, u_{s+1}=t_{s+1}, s \in\{-1,0,1, \ldots\}, n \geq s .
$$

$$
u_{n}=\frac{1}{1-p}+p^{n} C_{1}+(-p)^{n} C_{2} .
$$

Moreover, we obtain the relations (5) and (7) imply that

$$
t_{n+1}-u_{n+1} \leq p^{2}\left(t_{n-1}-u_{n-1}\right), n>s, p \in(0,1) .
$$

Thus we get

$$
t_{n} \leq u_{n}, n>s .
$$

Hence, we obtain from (7), (8) and Lemma 5,

$$
1<t_{n} \leq \frac{1}{1-p}+p^{n} C_{1}+(-p)^{n} C_{2}=M_{1},
$$


where

$$
\begin{aligned}
C_{1} & =\frac{1}{2 p}\left(p t_{0}+t_{1}-\frac{1+p}{1-p}\right), \\
C_{2} & =\frac{1}{2 p}\left(p t_{0}-t_{1}+1\right) .
\end{aligned}
$$

Likewise we have that

$$
1<z_{n} \leq \frac{1}{1-p}+p^{n} C_{3}+(-p)^{n} C_{4}=M_{2},
$$

where

$$
\begin{aligned}
C_{3} & =\frac{1}{2 p}\left(p z_{0}+z_{1}-\frac{1+p}{1-p}\right) \\
C_{4} & =\frac{1}{2 p}\left(p z_{0}-z_{1}+1\right)
\end{aligned}
$$

\subsection{Stability of System}

Now, we firstly consider a transformation such as:

$$
\left(t_{n}, t_{n-1}, \cdots, t_{n-m}, z_{n}, z_{n-1}, \cdots, z_{n-m}\right) \rightarrow\left(f, f_{1}, \cdots, f_{m}, g, g_{1}, \cdots, g_{m}\right)
$$

where $f=1+p \frac{z_{n}}{z_{n-m}^{2}}, f_{1}=t_{n}, \cdots f_{m}=t_{n-m}, g=1+p \frac{t_{n}}{t_{n-m}^{2}}, g_{1}=z_{n}, \cdots, g_{m}=z_{n-m}$. Hence we obtain the following the jacobian matrix about equilibrium point $(\bar{t}, \bar{z})$ :

$$
B(\bar{t}, \bar{z})=\left(\begin{array}{cccccccccc}
0 & 0 & \cdots & 0 & 0 & \frac{p}{\bar{z}^{2}} & 0 & \cdots & 0 & \frac{-2 p}{\bar{z}^{2}} \\
1 & 0 & \cdots & 0 & 0 & 0 & 0 & \cdots & 0 & 0 \\
\vdots & \vdots & \ddots & \vdots & \vdots & \vdots & \vdots & \ddots & \vdots & \vdots \\
0 & 0 & \cdots & 1 & 0 & 0 & 0 & \cdots & 0 & 0 \\
\frac{p}{t^{2}} & 0 & \cdots & 0 & \frac{-2 p}{t^{2}} & 0 & 0 & \cdots & 0 & 0 \\
0 & 0 & \cdots & 0 & 0 & 1 & 0 & \cdots & 0 & 0 \\
\vdots & \vdots & \ddots & \vdots & \vdots & \vdots & \vdots & \ddots & \vdots & \vdots \\
0 & 0 & \cdots & 0 & 0 & 0 & 0 & \cdots & 1 & 0
\end{array}\right)_{(2 m+2) \times(2 m+2)}
$$

Theorem 7 The positive equilibrium point $(\bar{t}, \bar{z})$ of system (4) is locally asymptotically stable.

Proof. The linearized system of system (4) about the positive equilibrium point is given by 
$X_{N+1}=B(\bar{t}, \bar{z}) X_{N}$, where

$$
\begin{aligned}
& X_{N}=\left(\begin{array}{c}
t_{n} \\
\vdots \\
t_{n-m} \\
z_{n} \\
\vdots \\
z_{n-m}
\end{array}\right), \\
& E=\left(\begin{array}{cccccccccc}
0 & 0 & \cdots & 0 & 0 & c & 0 & \cdots & 0 & -2 c \\
1 & 0 & \cdots & 0 & 0 & 0 & 0 & \cdots & 0 & 0 \\
\vdots & \vdots & \ddots & \vdots & \vdots & \vdots & \vdots & \ddots & \vdots & \vdots \\
0 & 0 & \cdots & 1 & 0 & 0 & 0 & \cdots & 0 & 0 \\
c & 0 & \cdots & 0 & -2 c & 0 & 0 & \cdots & 0 & 0 \\
0 & 0 & \cdots & 0 & 0 & 1 & 0 & \cdots & 0 & 0 \\
\vdots & \vdots & \ddots & \vdots & \vdots & \vdots & \vdots & \ddots & \vdots & \vdots \\
0 & 0 & \cdots & 0 & 0 & 0 & 0 & \cdots & 1 & 0
\end{array}\right)_{(2 m+2) \times(2 m+2)}
\end{aligned}
$$

and $c=\frac{p}{\bar{z}^{2}}=\frac{p}{t^{2}}$.

Let $\lambda_{1}, \lambda_{2}, \cdots, \lambda_{2 m+2}$ denote the $2 m+2$ eigenvalues of Matrix $E$. Let $D=\operatorname{diag}\left(d_{1}, d_{2}, d_{2 m+2}\right)$ be a diagonal matrix such that $d_{1}=d_{m+2}=1, d_{k}=d_{m+1+k}=$ $1-k \varepsilon, 1 \leq k \leq m$ and

$$
0<\varepsilon<\min \left\{\frac{1}{m+1}, \frac{1-3 c}{m+1}\right\} .
$$

Clearly, $D$ is invertible matrix. In computing matrix $D E D^{-1}$, we get that $D E D^{-1}=$

$$
\left(\begin{array}{ccccccccc}
0 & 0 & \cdots & 0 & 0 & c d_{1} d_{m+2}^{-1} & 0 & \cdots & 0 \\
d_{2} d_{1}^{-1} & 0 & \cdots & 0 & 0 & 0 & 0 & \cdots & 0 \\
\vdots & \vdots & \ddots & \vdots & \vdots & \vdots & \vdots & \ddots & \vdots \\
0 & 0 & \cdots & d_{m+1} d_{m}^{-1} & 0 & 0 & 0 & \cdots & 0 \\
c d_{m+2} d_{1}^{-1} & 0 & \cdots & 0 & -2 c d_{m+2} d_{m+1}^{-1} & 0 & 0 & \cdots & 0 \\
0 & 0 & \cdots & 0 & 0 & d_{m+3} d_{m+2}^{-1} & 0 & \cdots & 0 \\
\vdots & \vdots & \ddots & \vdots & \vdots & \vdots & \vdots & \ddots & \vdots \\
0 & 0 & \cdots & 0 & 0 & 0 & 0 & \cdots & d_{2 m+2} d_{2 m+1}^{-1}
\end{array}\right.
$$

From the following two inequalities

$$
\begin{aligned}
& 1=d_{1}>d_{2}>\cdots>d_{m}>d_{m+1}>0, \\
& 1=d_{m+2}>d_{m+3}>\cdots>d_{2 m+1}>d_{2 m+2}>0
\end{aligned}
$$

it implies that

$$
\begin{aligned}
d_{2} d_{1}^{-1} & <1, d_{3} d_{2}^{-1}<1, \cdots, d_{m+1} d_{m}^{-1}<1 \\
d_{m+3} d_{m+2}^{-1} & <1, d_{m+4} d_{m+3}^{-1}<1, \cdots, d_{2 m+2} d_{2 m+1}^{-1}<1
\end{aligned}
$$


Moreover we have that

$$
\begin{gathered}
c d_{1} d_{m+2}^{-1}+2 c d_{1} d_{2 m+2}^{-1}=c\left(1+\frac{2}{1-(m+1) \varepsilon}\right)<1, \\
c d_{m+2} d_{1}^{-1}+2 c d_{m+2} d_{m+1}^{-1}=c\left(1+\frac{2}{1-(m+1) \varepsilon}\right)<1 .
\end{gathered}
$$

It is a well-known fact that $E$ has the same eigenvalues as $D E D^{-1}$. Thus we get

$$
\begin{aligned}
\max _{1 \leq k \leq 2 m+2}\left|\lambda_{k}\right| & =\left\|D E D^{-1}\right\| \\
& =\max \left\{\begin{array}{c}
d_{2} d_{1}^{-1}, \cdots, d_{m+1} d_{m}^{-1}, \\
d_{m+3} d_{m+2}^{-1}, \cdots, d_{2 m+2} d_{2 m+1}^{-1}, \\
c d_{1} d_{m+2}^{-1}+2 c d_{1} d_{2 m+2}^{-1}, \\
c d_{m+2} d_{1}^{-1}+2 c d_{m+2} d_{m+1}^{-1}
\end{array}\right\} \\
& <1 .
\end{aligned}
$$

So, the positive equilibrium point $(\bar{t}, \bar{z})$ of system (4) is locally asymptotically stable.

Theorem 8 Suppose that $0<p<\frac{1}{2}$. Then the positive equilibrium point of system (4) is globally asymptotically stable.

Proof. From Theorem 6, we know that

$$
\begin{aligned}
& 1<l_{1}=\lim _{n \rightarrow \infty} t_{n} \leq M_{1}, \\
& 1<l_{2}=\lim _{n \rightarrow \infty} z_{n} \leq M_{2}, \\
& 1<L_{1}=\lim _{n \rightarrow \infty} t_{n} \leq M_{1}, \\
& 1<L_{2}=\lim _{n \rightarrow \infty} z_{n} \leq M_{2} .
\end{aligned}
$$

Thus we have the followings for system (4)

$$
\begin{aligned}
L_{1} & \leq 1+p \frac{L_{2}}{l_{2}^{2}}, l_{1} \geq 1+p \frac{l_{2}}{L_{2}^{2}}, \\
L_{2} & \leq 1+p \frac{L_{1}}{l_{1}^{2}}, l_{2} \geq 1+p \frac{l_{1}}{L_{1}^{2}} .
\end{aligned}
$$

Therefore we obtain

$$
\begin{aligned}
& L_{1}+p \frac{l_{1}}{L_{1}} \leq L_{1} l_{2} \leq l_{2}+p \frac{L_{2}}{l_{2}} \\
& L_{2}+p \frac{l_{2}}{L_{2}} \leq L_{2} l_{1} \leq l_{1}+p \frac{L_{1}}{l_{1}}
\end{aligned}
$$


Hence we get that

$$
\begin{aligned}
L_{1}+p \frac{l_{1}}{L_{1}}+L_{2}+p \frac{l_{2}}{L_{2}} & \leq l_{2}+p \frac{L_{2}}{l_{2}}+l_{1}+p \frac{L_{1}}{l_{1}}, \\
L_{1}+p \frac{l_{1}}{L_{1}}+L_{2}+p \frac{l_{2}}{L_{2}}-l_{2}-p \frac{L_{2}}{l_{2}}-l_{1}-p \frac{L_{1}}{l_{1}} & \leq 0, \\
\left(L_{1}-l_{1}\right)\left(1-p\left(\frac{1}{l_{1}}+\frac{1}{L_{1}}\right)\right)+\left(L_{2}-l_{2}\right)\left(1-p\left(\frac{1}{l_{2}}+\frac{1}{L_{2}}\right)\right) & \leq 0 .
\end{aligned}
$$

Now if $p \in\left(0, \frac{1}{2}\right)$ then

$$
\begin{aligned}
& 1-p\left(\frac{1}{l_{1}}+\frac{1}{L_{1}}\right)>0 \\
& 1-p\left(\frac{1}{l_{2}}+\frac{1}{L_{2}}\right)>0 .
\end{aligned}
$$

Therefore, we obtain that

So, $L_{1}=l_{1}$ and $L_{2}=l_{2}$.

$$
L_{1}-l_{1}=0, L_{2}-l_{2}=0
$$

\subsection{Rate of convergence of system (4)}

This section, we investigate the rate of convergence of system (4).

Theorem 9 Assume that $0<p<\frac{1}{2}$ and $\left\{\left(t_{n}, z_{n}\right)\right\}$ be a solution of the system (4) such that $\lim _{n \rightarrow \infty} t_{n}=\bar{t}$ and $\lim _{n \rightarrow \infty} z_{n}=\bar{z}$. Then the error vector

$$
E_{n}=\left(\begin{array}{c}
e_{n}^{1} \\
\vdots \\
e_{n-m}^{1} \\
e_{n}^{2} \\
\vdots \\
e_{n-m}^{2}
\end{array}\right)=\left(\begin{array}{c}
t_{n}-\bar{t} \\
\vdots \\
t_{n-m}-\bar{t} \\
z_{n}-\bar{z} \\
\vdots \\
z_{n-m}-\bar{z}
\end{array}\right)
$$

of every solution of system (4) satisfies both of the following asymptotic relations:

$$
\begin{aligned}
& \lim _{n \rightarrow \infty} \sqrt[n]{\left\|E_{n}\right\|}=\left|\lambda F_{J}(\bar{t}, \bar{z})\right|, \\
& \lim _{n \rightarrow \infty} \frac{\left\|E_{n+1}\right\|}{\left\|E_{n}\right\|}=\left|\lambda F_{J}(\bar{t}, \bar{z})\right|,
\end{aligned}
$$

where $\lambda F_{J}(\bar{t}, \bar{z})$ are the characteristic roots of the Jacobian matrix $F_{J}(\bar{t}, \bar{z})$. 
Proof. We firstly set to find the error terms,

$$
\begin{aligned}
t_{n+1}-\bar{t} & =\sum_{i=0}^{k} A_{i}\left(t_{n-i}-\bar{t}\right)+\sum_{i=0}^{k} B_{i}\left(z_{n-i}-\bar{z}\right), \\
z_{n+1}-\bar{z} & =\sum_{i=0}^{k} C_{i}\left(t_{n-i}-\bar{t}\right)+\sum_{i=0}^{k} D_{i}\left(z_{n-i}-\bar{z}\right),
\end{aligned}
$$

and $e_{n}^{1}=t_{n}-\bar{t}, e_{n}^{2}=z_{n}-\bar{z}$. Hence we obtain

$$
\begin{aligned}
e_{n+1}^{1} & =\sum_{i=0}^{k} A_{i} e_{n-i}^{1}+\sum_{i=0}^{k} B_{i} e_{n-i}^{2}, \\
e_{n+1}^{2} & =\sum_{i=0}^{k} C_{i} e_{n-i}^{1}+\sum_{i=0}^{k} D_{i} e_{n-i}^{2},
\end{aligned}
$$

where $A_{i}=0$ and $D_{i}=0$ for $i=0,1, \cdots, m$,

$$
\begin{aligned}
B_{0} & =\frac{p}{z_{n-m}^{2}}, B_{i}=0, i \in\{1,2, \cdots, m-1\}, B_{m}=\frac{-p\left(\bar{z}+z_{n-m}\right)}{\bar{z} z_{n-m}^{2}}, \\
C_{0} & =\frac{p}{t_{n-m}^{2}}, C_{i}=0, i \in\{1,2, \cdots, m-1\}, C_{m}=\frac{-p\left(\bar{t}+t_{n-m}\right)}{\bar{t} t_{n-m}^{2}} .
\end{aligned}
$$

Taking the limits, we have $\lim _{n \rightarrow \infty} A_{i}=\lim _{n \rightarrow \infty} D_{i}=0$ for $i \in\{0,1, \cdots, m\}$ and $\lim _{n \rightarrow \infty} B_{i}=\lim _{n \rightarrow \infty} C_{i}=0$ for $i \in\{1, \cdots, m-1\}$. Moreover we obtain that

$$
\begin{aligned}
\lim _{n \rightarrow \infty} B_{0} & =\frac{p}{\bar{z}^{2}}, \lim _{n \rightarrow \infty} B_{m}=\frac{-2 p}{\bar{z}^{2}}, \\
\lim _{n \rightarrow \infty} C_{0} & =\frac{p}{\overline{t^{2}}}, \lim _{n \rightarrow \infty} C_{m}=\frac{-2 p}{\bar{t}^{2}}
\end{aligned}
$$

Hence

$$
\begin{aligned}
& B_{0}=\frac{p}{\bar{z}^{2}}+a_{n}, B_{m}=\frac{-2 p}{\bar{z}^{2}}+b_{n}, \\
& C_{0}=\frac{p}{\bar{t}^{2}}+c_{n}, C_{m}=\frac{-2 p}{\bar{t}^{2}}+d_{n},
\end{aligned}
$$

where $a_{n} \rightarrow 0, b_{n} \rightarrow 0, c_{n} \rightarrow 0, d_{n} \rightarrow 0$ as $n \rightarrow \infty$. Hence, we get the system of the form (2)

where

$$
E_{n+1}=(A+B(n)) E_{n}
$$

$$
A=\left(\begin{array}{cccccccccc}
0 & 0 & \cdots & 0 & 0 & \frac{p}{\bar{z}^{2}} & 0 & \cdots & 0 & \frac{-2 p}{\bar{z}^{2}} \\
1 & 0 & \cdots & 0 & 0 & 0 & 0 & \cdots & 0 & 0 \\
\vdots & \vdots & \ddots & \vdots & \vdots & \vdots & \vdots & \ddots & \vdots & \vdots \\
0 & 0 & \cdots & 1 & 0 & 0 & 0 & \cdots & 0 & 0 \\
\frac{p}{t^{2}} & 0 & \cdots & 0 & \frac{-2 p}{t^{2}} & 0 & 0 & \cdots & 0 & 0 \\
0 & 0 & \cdots & 0 & 0 & 1 & 0 & \cdots & 0 & 0 \\
\vdots & \vdots & \ddots & \vdots & \vdots & \vdots & \vdots & \ddots & \vdots & \vdots \\
0 & 0 & \cdots & 0 & 0 & 0 & 0 & \cdots & 1 & 0
\end{array}\right)
$$




$$
B(n)=\left(\begin{array}{cccccccccc}
0 & 0 & \cdots & 0 & 0 & a_{n} & 0 & \cdots & 0 & b_{n} \\
1 & 0 & \cdots & 0 & 0 & 0 & 0 & \cdots & 0 & 0 \\
\vdots & \vdots & \ddots & \vdots & \vdots & \vdots & \vdots & \ddots & \vdots & \vdots \\
0 & 0 & \cdots & 1 & 0 & 0 & 0 & \cdots & 0 & 0 \\
c_{n} & 0 & \cdots & 0 & d_{n} & 0 & 0 & \cdots & 0 & 0 \\
0 & 0 & \cdots & 0 & 0 & 1 & 0 & \cdots & 0 & 0 \\
\vdots & \vdots & \ddots & \vdots & \vdots & \vdots & \vdots & \ddots & \vdots & \vdots \\
0 & 0 & \cdots & 0 & 0 & 0 & 0 & \cdots & 1 & 0
\end{array}\right)
$$

and $\|B(n)\| \rightarrow 0$ as $n \rightarrow \infty$. Therefore, we can write the limiting system of error terms about the equilibrium point $(\bar{t}, \bar{z})$ as follows:

$$
\left(\begin{array}{c}
e_{n+1}^{1} \\
e_{n}^{1} \\
\vdots \\
e_{n-m+1}^{1} \\
e_{n+1}^{2} \\
e_{n}^{2} \\
\vdots \\
e_{n-m+1}^{2}
\end{array}\right)=\left(\begin{array}{cccccccccc}
0 & 0 & \cdots & 0 & 0 & \frac{p}{\bar{z}^{2}} & 0 & \cdots & 0 & \frac{-2 p}{\bar{z}^{2}} \\
1 & 0 & \cdots & 0 & 0 & 0 & 0 & \cdots & 0 & 0 \\
\vdots & \vdots & \ddots & \vdots & \vdots & \vdots & \vdots & \ddots & \vdots & \vdots \\
0 & 0 & \cdots & 1 & 0 & 0 & 0 & \cdots & 0 & 0 \\
\frac{p}{t^{2}} & 0 & \cdots & 0 & \frac{-2 p}{t^{2}} & 0 & 0 & \cdots & 0 & 0 \\
0 & 0 & \cdots & 0 & 0 & 1 & 0 & \cdots & 0 & 0 \\
\vdots & \vdots & \ddots & \vdots & \vdots & \vdots & \vdots & \ddots & \vdots & \vdots \\
0 & 0 & \cdots & 0 & 0 & 0 & 0 & \cdots & 1 & 0
\end{array}\right)\left(\begin{array}{c}
e_{n}^{1} \\
e_{n-1}^{1} \\
\vdots \\
e_{n-m}^{1} \\
e_{n}^{2} \\
e_{n-1}^{2} \\
\vdots \\
e_{n-m}^{2}
\end{array}\right),
$$

which is same as linearized system of system (4) about equilibrium point $(\bar{t}, \bar{z})$.

\subsection{Oscillatory of solutions of system (4)}

We now study the oscillatory of solutions of system(4).

Theorem 10 Suppose that $\left\{\left(t_{n}, z_{n}\right)\right\}$ be a positive solution of system (4) and $p>0$. Then, either $\left\{\left(t_{n}, z_{n}\right)\right\}$ solution of system (4) has a single semicycle or $\left\{\left(t_{n}, z_{n}\right)\right\}$ solution of system (4) has semicycles with at most $m$ terms.

Proof. Let $\left\{\left(t_{n}, z_{n}\right)\right\}$ solution of system (4) has at least two semicycles. Hence there exists $N \geq 0$ such that either

or

$$
t_{N}, z_{N+1}<\bar{t}=\bar{z}<t_{N+1}, z_{N}
$$

$$
t_{N+1}, z_{N}<\bar{t}=\bar{z}<t_{N}, z_{N+1} .
$$

Firstly we assume that $t_{N}, z_{N+1}<\bar{t}=\bar{z}<t_{N+1}, z_{N}$. Moreover, we suppose that positive semicycle have $m$ terms and it begins with the term $\left(t_{N+1}, z_{N+1}\right)$. Thus we obtain the followings

$$
\begin{aligned}
t_{N} & <\bar{t}=\bar{z}<t_{N+m}, \\
z_{N+m} & <\bar{t}=\bar{z}<z_{N} .
\end{aligned}
$$


From this we get

$$
\begin{aligned}
& t_{N+m+1}=1+p \frac{z_{N+m}}{z_{N}^{2}}<\bar{t}=\bar{z} \\
& z_{N+m+1}=1+p \frac{t_{N+m}}{t_{N}^{2}}>\bar{t}=\bar{z}
\end{aligned}
$$

\section{Numerical examles}

In this here, we provide two examples to verify our theoretical results.

Example 1 We handle system (4) with $m=3$ and $p=0.49$. Therefore we obtain the following fourth order system of difference equations

$$
t_{n+1}=1+0.49 \frac{z_{n}}{z_{n-3}^{2}}, z_{n+1}=1+0.49 \frac{t_{n}}{t_{n-3}^{2}} .
$$

Now we consider this system with the initial values $t_{-3}=6, t_{-2}=1, t_{-1}=0.8, t_{0}=$ $4, z_{-3}=0.4, z_{-2}=5, z_{-1}=3$ and $z_{0}=10$. Thus positive equilibrium point $(\bar{t}, \bar{z})=$ $(1.36,1.36)$ of system (9) is globally asymptotically stable. Figure 1 verifies to our theoretical results.

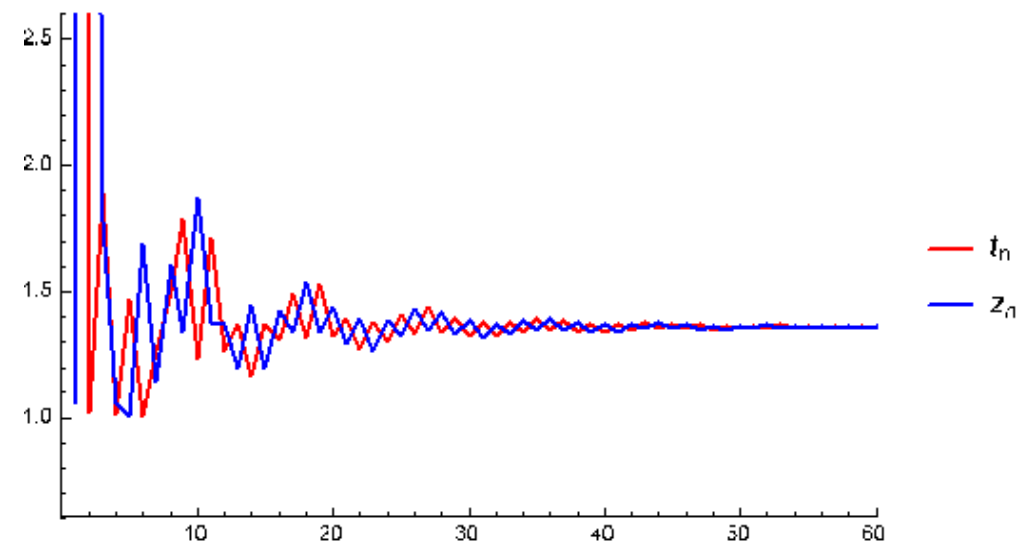

1. Plot of system (4) with $m=3$ and $p=0.49$.

Example 2 We take system (4) with $m=2$ and $p=1.5$. Hence we have the following third order system of difference equations

$$
t_{n+1}=1+1.5 \frac{z_{n}}{z_{n-2}^{2}}, z_{n+1}=1+1.5 \frac{t_{n}}{t_{n-2}^{2}} .
$$


We also consider this system with the initial values $t_{-2}=3, t_{-1}=4, t_{0}=0.6, z_{-2}=$ $2, z_{-1}=0.4$ and $z_{0}=3$. Thus the solutions of system (10) oscillate about positive equilibrium point $(\bar{t}, \bar{z})=(1.82,1.82)$. Figure 2 verifies our theoretical results.

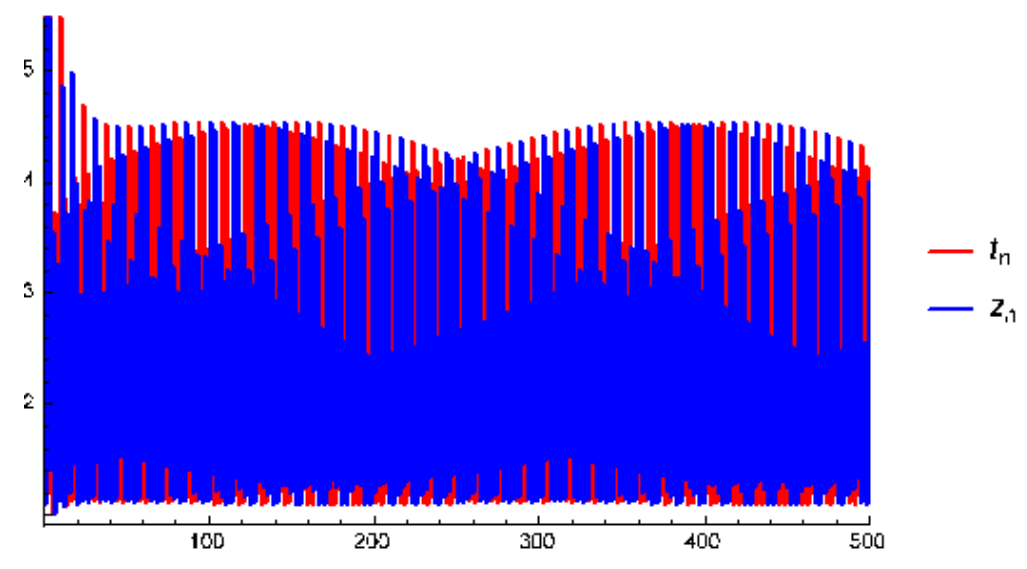

2. Plot of system (4) with $m=2$ and $p=1.5$.

\section{Conclusions and an Open Problem}

In this study, we analysis the dynamics of system (4) of higher order difference equations with quadratic terms. We first examine the equilibrium point of related system. Then we investigate the existence of bounded solutions of system (4). We also study the stability analysis of solutions of system (4). As a result of this, we obtain that positive equilibrium point of system (4) is globally asymptotically stable when $0<p<\frac{1}{2}$. Additionally we investigate the rate of convergence of system (4). Moreover we have an oscillatory result such that the solution of system (4) has a single semicycle or the solution of system (4) has semicycles with at most $m$ terms. In addition we give two numerical examles to verify our theoretical results.

Open Problem: Investigate the dynamics of following non-symmetric system of difference equations

$$
x_{n+1}=A+B \frac{y_{n}}{y_{n-m}^{2}}, y_{n+1}=C+D \frac{x_{n}}{x_{n-m}^{2}}, n=0,1, \ldots,
$$

where the initial values are real numbers and both $A \neq B$ and $C \neq D$ are real numbers.

\section{References}

[1] Agarwal, R.P., Wong, P.J., Advanced topics in difference equations (Vol. 404), Springer Science \& Business Media, (2013). 
[2] Bacani, J.B., Rabago, J.F.T., On two nonlinear difference equations. Dyn. Contin. Discrete Impuls. Syst. Ser. A Math. Anal., 24, 375-394, 2017.

[3] Bešo, E., Kalabušić, S., Mujić, N. Pilav E. Boundedness of solutions and stability of certain second-order difference equation with quadratic term. Adv. Differ. Equ. 2020, 19, 1-22 (2020). https://doi.org/10.1186/s13662-019-2490-9

[4] Bilgin, A., Kulenovic, M.R.S., Global asymptotic stability for discrete single species population models, Discrete Dyn. Nat. Soc., 2017, 1-15 (2017).

[5] Burgić, Dž., Kulenović, M.R.S., Nurkanović, M. Global dynamics of a rational system of difference equations in the plane. Commun. Appl. Nonlinear Anal. 15(1), 71-84 (2008).

[6] Camouzis, E., Papaschinopoulos, G., Global asymptotic behavior of positive solutions on the system of rational difference equations $x_{n+1}=1+x_{n} / y_{n-m}, y_{n+1}=$ $1+y_{n} / x_{n-m}$, Appl. Math. Lett. 17(6), 733-737 (2004).

[7] Din, Q.: Asymptotic behavior of an anti-competitive system of second-order difference equations. J. Egyptian Math. Soc.. 24(1), 37-43 (2016).

[8] Elabbasy, E.M., Eleissawy, S.M. Asymptotic behavior of two dimensional rational system of difference equations. Dyn. Contin. Discrete Impuls. Syst. Ser. B Appl. Algorithms, 2013, 20: 221-235.

[9] Elaydi, S. An Introduction to Difference Equations, Springer-Verlag, New York, Inc, 1996.

[10] Gümüs M., The global asymptotic stability of a system of difference equations, J. Difference Equ. Appl. 24:6, 976-991 (2018) , DOI: 10.1080/10236198.2018.1443445

[11] Hadžiabdić, V., Kulenović, M.R.S., Pilav, E. Dynamics of a two-dimensional competitive system of rational difference equations with quadratic terms. Adv. Differ. Equ. 2014, 301, 1-32, (2014). https://doi.org/10.1186/1687-1847-2014-301

[12] Khan, A.Q., Qureshi, M.N. Qualitative behavior of two systems of higher-order difference equations. Math. Methods Appl. Sci. 39(11), 3058-3074, (2016).

[13] Khan, A.Q., Sharif, K. Global dynamics, forbidden set and transcritical bifurcation of a one-dimensional discrete-time laser model. Math. Meth. Appl. Sci. 2020; 1-13. https://doi.org/10.1002/mma.6201

[14] Kulenovic, M.R.S., Nurkanovic, M., Nurkanovic, Z.Global dynamics of certain mix monotone difference equation via center manifold theory and theory of monotone maps, Sarajevo J. Math., 15(2), 129-154, (2019) .

[15] Kulenovic, M. R., \& Ladas, G. (2001). Dynamics of second order rational difference equations: with open problems and conjectures. Chapman and Hall/CRC.

[16] Kocic, V. L., and Gerasimos Ladas. Global behavior of nonlinear difference equations of higher order with applications. Vol. 256. Springer Science \& Business Media, 1993.

[17] Okumuş, İ., Soykan, Y.: Dynamical behavior of a system of three-dimensional nonlinear difference equations. Adv. Differ. Equ. 2018:224, 1-15 (2018).

[18] Papaschinopoulos G, Schinas CJ. On the system of two nonlinear difference equations $x_{n+1}=A+x_{n-1} / y_{n}, y_{n+1}=A+y_{n-1} / x_{n}$. Int. J. Math. Math. Sci. 2000; 23(12):839-848. 
[19] Papaschinopoulos G. Schinas C.J., On a system of two nonlinear difference equations, J. Math. Anal. Appl. 219, 415-426, (1998)

[20] Pituk, M.: More on Poincare's and Perron's theorems for difference equations. J. Difference Equ. Appl. 8, 201-216 (2002).

[21] Simşek, D, B Oğul, and C Çinar. Solution of the rational difference equation $\mathrm{xn}+1=$ xn-17/1+ xn-5• xn-11." Filomat 33.5 (2019): 1353-1359.

[22] Qureshi, S.M., Khan, A.Q.: Global Dynamics of a 3x6 System of Difference Equations. Discrete Dyn. Nat. Soc. 2019, 1-14 (2019).

[23] Taşdemir, E. On the Global Asymptotic Stability of a Two Dimensional System of Difference Equations with Quadratic Terms. Preprints 2020, 2020090609 (doi: 10.20944/preprints202009.0609.v1).

[24] Yang, L., Yang, J., Dynamics of a system of two nonlinear difference equations. Int. J. Contemp. Math. Sciences, 6(5): 209-214, 2011.

[25] Zhang D, Ji W, Wang L, Li X. On the Symmetrical System of Rational Difference Equation $x_{n+1}=A+y_{n-k} / y_{n}, y_{n+1}=A+x_{n-k} / x_{n}$. Applied Mathematics. 4(5), 834-837, 2013.

[26] Zhang Y, Yang X, Evans DJ, Zhu C. On the nonlinear difference equation system $x_{n+1}=A+x_{n-m} / y_{n}, y_{n+1}=A+y_{n-m} / x_{n}$. Comput. Math. Appl. 53, 1561-1566, 2007. 